\title{
Antimicrobial solid media for screening nonsterile Arabidopsis thaliana seeds
}

Behrendorff, James; Borràs I Gas, Guillem; Pribil, Mathias

Published in:

Physiologia Plantarum

DOI:

10.1111/ppl.13079

Publication date:

2020

Document version

Publisher's PDF, also known as Version of record

Document license:

CC BY

Citation for published version (APA):

Behrendorff, J., Borràs I Gas, G., \& Pribil, M. (2020). Antimicrobial solid media for screening nonsterile Arabidopsis thaliana seeds. Physiologia Plantarum. https://doi.org/10.1111/ppl.13079 


\title{
Antimicrobial solid media for screening non-sterile Arabidopsis thaliana seeds
}

\author{
James B.Y.H. Behrendorff*, (1) Guillem Borràs-Gas (1) and Mathias Pribil (10 \\ Copenhagen Plant Science Centre, Department of Plant and Environmental Sciences, University of Copenhagen, Frederiksberg, Denmark
}

\author{
Correspondence \\ *Corresponding author, \\ e-mail: james@buildwithbio.science
}

Received 1 December 2019;

revised 14 February 2020

doi:10.1111/ppl.13079

\begin{abstract}
Stable genetic transformation of plants is a low-efficiency process, and identification of positive transformants usually relies on screening for expression of a co-transformed marker gene. Often this involves germinating seeds on solid media containing a selection reagent. Germination on solid media requires surface sterilization of seeds and careful aseptic technique to prevent microbial contamination, but surface sterilization techniques are time consuming and can cause seed mortality if not performed carefully. We developed an antimicrobial cocktail that can be added to solid media to inhibit bacterial and fungal growth without impairing germination, allowing us to bypass the surface sterilization step. Adding a combination of terbinafine $(1 \mu \mathrm{M})$ and timentin $\left(200 \mathrm{mg} \mathrm{l}^{-1}\right)$ to Murashige and Skoog agar delayed the onset of observable microbial growth and did not affect germination of non-sterile seeds from 10 different wild-type and mutant Arabidopsis thaliana accessions. We named this antimicrobial solid medium "MSTT agar". Seedlings sown in non-sterile conditions could be maintained on MSTT agar for up to a week without observable contamination. This medium was compatible with rapid screening methods for hygromycin B, phosphinothricin (BASTA) and nourseothricin resistance genes, meaning that positive transformants can be identified from non-sterile seeds in as little as 4 days after stratification, and transferred to soil before the onset of visible microbial contamination. By using MSTT agar we were able to select genetic transformants on solid media without seed surface sterilization, eliminating a tedious and timeconsuming step.
\end{abstract}

\section{Introduction}

Driven by cheap and reliable methods of DNA assembly, the synthetic biology revolution has made it possible for molecular biologists to design and build dozens of new plasmids in as little as 1 or 2 weeks even without automation equipment. Plant science has not fully exploited these advances in molecular cloning to the same extent as other disciplines, partly because of experimental throughput limitations unique to plants.
Agrobacterium-mediated genetic transformation is one of the most versatile and accessible methods for modifying the genome of Arabidopsis thaliana (Bechtold and Pelletier 1998, Zhang et al. 2006), but this approach produces only a small minority of seeds in the $\mathrm{T}_{1}$ generation that carry the transgene of interest. Transformation efficiencies between 0.57 and $2.57 \%$ have been reported with optimized variations of the classic floral dipping method (Chung et al. 2000, Martinez-Trujillo

Abbreviations - BASTA, phosphinothricin or glufosinate; mEGFP, monomeric enhanced green fluorescent protein; MS, Murashige and Skoog medium; MSTT, Murashige and Skoog medium with terbinafine and timentin; MSTT+SUC, MSTT with sucrose; PDS3, phytoene desaturase 3. 
et al. 2004, Zhang et al. 2006). Identifying this minority of positive transformants usually relies on selection or screening for a co-transformed marker gene.

The most common selection approaches involve germinating seeds on an agar-based solid nutrient medium that contains a chemical reagent to select seedlings that express the corresponding marker gene. Popular selectable markers confer resistance to phosphinothricin (BASTA, also known as glufosinate), kanamycin, hygromycin B, or nourseothricin (also known as streptothricin) (Jelenska et al. 2000, Harrison et al. 2006). Germination on solid media requires that seeds are surface sterilized to prevent overgrowth by microbial contaminants during the selection process.

At the time when these screening methods were established, molecular cloning was a bottleneck in the experimental workflow of transgenic plant preparation and typically few transgenic lines were prepared simultaneously. This is no longer the case, yet the same screening methods are still widely used. Screening for successful stable transfection events now represents a significant bottleneck, especially when an experiment involves several different genetic designs.

The seed sterilization step in particular has disadvantages that become more pronounced when screening increasing numbers of transformant lines. Liquid sterilization in hypochlorite bleach has a low seed mortality rate but is tedious, requiring several washing steps that become time consuming when preparing large quantities of seeds (Lindsey et al. 2017). Chlorine gas is suitable for sterilizing seeds from multiple lines simultaneously, but gas sterilization still requires up to $4 \mathrm{~h}$ of waiting time and can have a relatively high mortality rate even when the gas concentration is carefully controlled (Lindsey et al. 2017). Mortality caused by the sterilization process could result in the loss of rare transformants or a reduction in the diversity of mutant libraries. Furthermore, surface sterilization does not necessarily eliminate microbial spores that can be trapped inside the seed coat during embryogenesis (Andargie and Li 2016).

We aimed to develop a method that would allow us to avoid surface sterilization of seeds altogether. Our approach was to identify a combination of antifungal and antibacterial compounds that inhibit microbial growth but do not impair Arabidopsis germination and growth. The antimicrobial medium presented here (MSTT agar) substantially delays microbial contamination. When combined with established rapid selection methods (Harrison et al. 2006), we were able to identify positive transformants from non-sterile seeds in less than 1 week and transfer them to soil for growth and propagation prior to the onset of observable microbial contamination.

\section{Materials and methods}

\section{Chemicals}

Murashige and Skoog medium including vitamins (MS medium) (Murashige and Skoog 1962) (Cat. no. M0222), hygromycin B (Cat. no. H0192) and timentin (ticarcillin 2NA and clavulanate K 15:1 mixture, Cat. no. T0190) were purchased from Duchefa Biochemie. Nourseothricin was purchased from Jena Bioscience (Cat. no. AB-102L). BASTA was purchased from Bayer Cropscience (Product no. 84442615). Terbinafine (Cat. no. T8826) was purchased from Merck. All other chemicals were the highest quality locally available. Stock solutions were prepared as follows: timentin, $200 \mathrm{mg} \mathrm{ml}^{-1}$ in water; nourseothricin, $50 \mathrm{mg} \mathrm{m}^{-1}$ in water; terbinafine, $1 \mathrm{mM}$ in dimethylsulfoxide (DMSO); carbenicillin, $50 \mathrm{mg} \mathrm{ml}^{-1}$ in water; BASTA, $50 \mathrm{mM}$ in water. Hygromycin B was used directly from the liquid stock provided by the manufacturer $\left(400 \mathrm{mg} \mathrm{m}^{-1}\right.$ in water).

\section{Plant accessions}

Arabidopsis thaliana mutants (curt1abcd (Armbruster et al. 2013), atpC1 (Dal Bosco et al. 2004), hcf136 (Meurer et al. 1998), pam68 (Armbruster et al. 2010), psal (Lunde et al. 2000), and npq4 (Li et al. 2000) and wildtype ecotypes (Columbia (Col-0), Landsberg erecta (Ler0 ), Wassilewskija (Ws-0), and Nossen (No-0)) were a gift from Prof. Dario Leister, Ludwig Maximilians Universität, Germany. Nicotiana tabacum (cv. Petit Havana) seeds were a gift from Dr. Lars Scharff (University of Copenhagen, Denmark).

\section{Antimicrobial compound screening}

MS agar $(0.5 \times)$ was prepared by dissolving Murashige and Skoog medium in water and adjusting the $\mathrm{pH}$ to 5.7 with $1 \mathrm{M}$ potassium hydroxide. Agar was added to a final concentration of $1 \%(\mathrm{w} / \mathrm{v})$ and sterilized by autoclaving. Sucrose was added from a filter-sterilized stock solution $(40 \% \mathrm{w} / \mathrm{v}$ in water). Antimicrobial reagents were added from the stock solutions described above (in see Chemicals), and all agar plates were poured in non-sterile conditions on a standard laboratory bench. In all screening experiments, non-sterile seeds were sown directly onto solidified agar without any pre-treatment. Throughout this study, seeds were stratified by wrapping the agar plates in aluminum foil and storing at $4^{\circ} \mathrm{C}$ for $68 \mathrm{~h}$. After stratification, agar plates were maintained under long day conditions $\left(16 \mathrm{~h}\right.$ light at $100 \mu \mathrm{mol} \mathrm{m} \mathrm{m}^{-2} \mathrm{~s}^{-1}, 8 \mathrm{~h}$ darkness, $22^{\circ} \mathrm{C}, 60 \%$ humidity) in a Fitotron SGC 120 growth chamber (Weisstechnik, Germany). 
During the development phase of this study we compared the effects of different antimicrobial reagents on germination of 10 different $A$. thaliana lines and N. tabacum. Therefore, for each condition tested there were $\mathrm{n}=11$ paired comparisons between treated and untreated seeds. On each plate, between 23 and 122 seeds were sown.

The final antimicrobial solid medium developed in this study (MSTT agar) contained 0.5× MS medium ( $\mathrm{pH} 5.7$ ), terbinafine $(1 \mu \mathrm{M})$, timentin $\left(200 \mathrm{mg} \mathrm{l}^{-1}\right)$, and agar $\left(10 \mathrm{~g} \mathrm{I}^{-1}\right)$. MSTT+suc agar also contained sucrose $(1 \%, w / v)$.

\section{Plasmids}

All cloning strategies were designed with Geneious 10.2.6 (http://www.geneious.com) and performed using the general principles of the Gibson assembly method (Gibson et al. 2009). Cartoon representations of plasmids were generated with Pigeon (Bhatia and Densmore 2013) (http://pigeon.synbiotools.org). Oligonucleotide primers and sources of template DNA are listed online in Appendix S2. Fluorescent reporter protein constructs: in a previous study (Behrendorff et al. 2019), a nourseothricin-selectable plasmid was prepared by replacing the bialaphos resistance (bar) gene from plasmid pB2GW7 (Karimi et al. 2002) with the nourseothricin acetyl transferase (nat) gene from Streptomyces noursei. The resulting plasmid is described as $\mathrm{pN} \_35 \mathrm{~S}$. The $\mathrm{ccdB}$ counter-selectable marker was replaced with the coding sequences for either mEGFP or mApple fluorescent proteins, placing fluorescent protein expression under the control of the cauliflower mosaic virus $35 \mathrm{~S}$ promoter (plasmids pN_35S/mEGFP and pN_35S/mApple are available at www.addgene.org as plasmids \#132565 [RRID:Addgene_132 565] and \#132566 [RRID: Addgene_132 566], respectively). The pB_35S/mEGFP plasmid was prepared by cloning the mEGFP coding sequence directly into the pB2GW7 backbone, replacing the $\mathrm{ccdB}$ counterselectable marker (www.addgene.org, plasmid \#135320 [RRID:Addgene_135 320]). We have also made available a hygromycin-selectable mEGFP expression plasmid, pH_35S/mEGFP (www.addgene. org, plasmid \#135321 [RRID:Addgene_135 321]), prepared by cloning the mEGFP coding sequence into the pH2GW7 (Karimi et al. 2002) backbone. Plasmid pN_35S/CTP-mCitrine was produced in an earlier study (Behrendorff et al. 2019) and encodes an mCitrine fluorescent protein fused in-frame to the chloroplast transit peptide from RuBisCO small subunit 1A (www. addgene.org, plasmid \#117989 [RRID: Addgene_117 989]). CRISPR constructs: an mApple fluorescent protein was fused in-frame to the C-terminus of a Streptomyces pyogenes Cas9 via a GGGGS flexible linker. The Cas9-mApple coding sequence and a PDS3 sgRNA under the control of the $A$. thaliana $U 6$ polymerase III promoter ( $\mathrm{Li}$ et al. 2013) were cloned into the pH2GW7 (Karimi et al. 2002) backbone (hygromycin selection). A previously-described promoter made by combining two $A$. thaliana egg cell specific promoters (Wang et al. 2015b) was then inserted upstream of the Cas9-mApple coding sequence to create GS2.1/EC (www.addgene.org, plasmid \#132568 [RRID: Addgene_132 568]).

Plasmids were transformed into Agrobacterium fabrum strain GV3101 (previously known as Agrobacterium tumefaciens GV3101 [Gan and Savka 2018) via electroporation with the following conditions: voltage $2500 \mathrm{~V}$, capacitance $25 \mu \mathrm{F}$, resistance $400 \Omega, 2 \mathrm{~mm}$ cuvette.

\section{Genetic transformation and screening}

A. thaliana (Col-0) was grown under long day conditions (16 h light at $100 \mu \mathrm{mol} \mathrm{m}{ }^{-2} \mathrm{~s}^{-1}, 8 \mathrm{~h}$ darkness) at $22^{\circ} \mathrm{C}$ and $60 \%$ humidity, and transformed according to a modified floral dip method described previously (MartinezTrujillo et al. 2004). Seeds collected from transformed plants were sown on MSTT or MSTT+suc agar with appropriate selection reagents.

Plants resistant to nourseothricin $\left(50 \mathrm{mg}^{-1}\right)$ or hygromycin B (15 $\left.\mathrm{mg} \mathrm{l}^{-1}\right)$ were identified by rapid screening for hypocotyl elongation (Harrison et al. 2006). After stratification, plates were shifted to growth chamber conditions and exposed to light for $6 \mathrm{~h}$. Plates were then wrapped in foil to maintain darkness for 2 full days and stored at $22^{\circ} \mathrm{C}$. On the fourth day, plates were unwrapped and resistant seedlings with elongated hypocotyls were clearly distinguishable from non-resistant seedlings. Plants resistant to BASTA $(50 \mu \mathrm{M})$ were identified by rapid screening for green expanded cotyledons (Harrison et al. 2006). Plates were shifted to growth chamber conditions after stratification and exposed to light for $6 \mathrm{~h}$, and then wrapped in foil to maintain darkness for 3 full days. Following the dark treatment, plates were unwrapped and kept in a growth chamber under long day conditions. Positive transformants could be identified 2 days later (i.e. the fifth day post-stratification), and differences between resistant and non-resistant seedlings were more pronounced on the sixth day poststratification.

In the case of plants transformed with fluorescent protein expression constructs ( $\mathrm{pN}$ _35S/mEGFP, pN_35S/ CTP-mCitrine, pN_35S/mApple, or pB_35S/mEGFP), transformation was verified by fluorescence imaging on a Bio-Rad ChemiDoc XRS+ (Bio-Rad Laboratories, Inc.). Green and yellow fluorescent signals (from mEGFP and 
chloroplast-targeted mCitrine) were captured using blue light epi-illumination and a $530 \mathrm{~nm}$ filter $(28 \mathrm{~nm}$ bandpass). Red fluorescence from mApple was captured with green light epi illumination and a $605 \mathrm{~nm}$ filter $(50 \mathrm{~nm}$ bandpass). For red fluorescence imaging, it was necessary to image seedlings on the fourth day post-stratification, prior to greening of cotyledons (i.e. on the same day that the germinating seedlings were removed from dark treatment). It was not possible to identify mApple fluorescence in green leaves due to interference from chlorophyll autofluorescence.

Homozygous pds3 mutants were identified by their distinct albino phenotype and were confirmed via Sanger sequencing (oligonucleotide primer details included online in Appendix S2).

\section{PCR from leaf tissue}

Diagnostic PCRs, preparative PCRs for Sanger sequencing, and PCRs to prepare A. thaliana DNA for cloning were performed using leaf tissue as the source of template DNA. A portion of leaf tissue (approximately $5 \mathrm{~mm}^{2}$ ) was homogenized by grinding in $50 \mu \mathrm{l}$ of $1 \times \mathrm{Q} 5$ reaction buffer (New England Biolabs Cat. No. B9027S) in a $1.5 \mathrm{ml}$ microcentrifuge tube with a micropestle. The homogenate was heated to $98^{\circ} \mathrm{C}$ for $10 \mathrm{~min}$, then cooled on ice. After cooling, leaf debris was separated by centrifugation $(30 \mathrm{~s}, 13000 \mathrm{~g})$. The supernatant was used directly as a source of template DNA $(1 \mu$ l template DNA per PCR).

\section{Results}

\section{Terbinafine as an antifungal reagent}

Terbinafine is an antifungal reagent that inhibits squalene epoxidase, causing a deficiency in the membrane lipid ergosterol (Ryder 1992). Squalene epoxidation is also a key step in the biosynthesis of plant sterols, and squalene epoxidase knockout mutants of $A$. thaliana Col-0 exhibit increased sensitivity toward terbinafine while wild-type plants appear phenotypically normal post-germination (Laranjeira et al. 2015). We sought to test whether low concentrations of terbinafine could be used to inhibit fungal growth without impairing germination of $A$. thaliana.

In a preliminary experiment, non-sterile seeds from four wild-type $A$. thaliana ecotypes (Columbia (Col-0), Landsberg erecta (Ler-0), Wassilewskija (Ws-0), and Nossen (No-0)), six photosynthetic gene mutants [curt1abcd (Armbruster et al. 2013), atpC1 (Dal Bosco et al. 2004), hcf136 (Meurer et al. 1998), pam68 (Armbruster et al. 2010), psaL (Lunde et al. 2000), and npq4 (Li et al. 2000)], and Nicotiana tabacum (cv. Petit Havana) were sown directly onto $0.5 \times$ Murashige and Skoog
(MS) agar with sucrose $(1 \%, \mathrm{w} / \mathrm{v})$ and terbinafine (added to a final concentration of $1,0.1$, or $0.01 \mu \mathrm{M}$ ). Negative control plates contained DMSO $(0.1 \%, \mathrm{v} / \mathrm{v})$ without terbinafine. While sucrose is not necessary for germination of wild-type plants, many mutants with impaired photosynthesis benefit from the addition of sucrose during germination. Sucrose also increases the risk of microbial contamination because it is a utilizable carbon source for most fungi and many bacteria. Therefore, we included sucrose in our media to ensure that our antimicrobial medium would be useful in cases where the inclusion of sucrose is necessary.

The onset of germination (defined as the first cotyledons to emerge on each plate) was determined by visual inspection and was scored qualitatively, as was the emergence of observable microbial contamination. Plates were inspected twice per day for 7 days (168 $\mathrm{h}$ in total).

Terbinafine did not affect the onset of germination at any of the concentrations tested (up to $1 \mu \mathrm{M}$ ) (online in Fig. S1A). In negative control agar plates that lacked terbinafine, microbial contamination was observed as early as $24 \mathrm{~h}$ after being transferred to growth chamber conditions (median time to visible contamination: $64 \mathrm{~h}$ ) (Fig. S1B). In the presence of terbinafine, there was a general trend toward delayed onset of microbial contamination with increasing terbinafine concentration. At $1 \mu \mathrm{M}$ terbinafine, all plates were free of microbial contamination after $168 \mathrm{~h}$ except for one plate, where a contaminant emerged after $112 \mathrm{~h}$. It was not obvious whether the contaminant was bacterial or fungal, but these results indicate that $1 \mu \mathrm{M}$ terbinafine decreases the likelihood of fungal contamination and/or delays fungal growth.

\section{Combining terbinafine with antibacterial $\beta$-lactam antibiotics}

$\beta$-lactam antibiotics were tested as the antibacterial reagent because they inhibit peptidoglycan biosynthesis in prokaryotes, whereas most other classes of prokaryote-targeting antibiotics also interfere with plastid (Ellis 1969, Mulo et al. 2003) and mitochondrial protein synthesis (Wang et al. 2015a). We examined the effects of carbenicillin and timentin, which are both commonly used for eliminating Agrobacteria from plant tissue culture (Lin et al. 1995, Zhang et al. 2006, Yan et al. 2015). Timentin is a mixture containing a $\beta$-lactam antibiotic (ticarcillin) and a $\beta$-lactamase inhibitor (clavulanic acid).

Timentin $\left(200 \mathrm{mg} \mathrm{l}^{-1}\right)$ or carbenicillin $\left(500 \mathrm{mg} \mathrm{l}^{-1}\right)$ was added to $0.5 \times \mathrm{MS}$ agar that contained sucrose $(1 \%$, $\mathrm{w} / \mathrm{v})$ and terbinafine $(1 \mu \mathrm{M})$. Non-sterile seeds were sown directly onto agar plates and stratified as described above, and then transferred to growth chambers and 
monitored by visual inspection. Germination was quantified by recording the number of germinated seedlings twice per day for the first 4 days, and once per day thereafter. Microbial contamination was recorded qualitatively.

The combination of terbinafine $(1 \mu \mathrm{M})$ and timentin (200 $\mathrm{mg} \mathrm{I}^{-1}$ ) did not inhibit germination of wild-type A. thaliana ecotypes or $N$. tabacum compared with untreated seeds (sown on $0.5 \times \mathrm{MS}$ agar + sucrose without antimicrobial additives) (Fig. 1). Photosynthetic mutant A. thaliana lines were also unaffected (Fig. 2) except in the case of the psaL mutant, where the inclusion of timentin may have impaired germination in $7 \%$ of seeds (at $168 \mathrm{~h}: 96 \%$ of untreated seeds had germinated vs $89 \%$ of seeds sown on timentin plus terbinafine).
Carbenicillin (500 $\mathrm{mg} \mathrm{I}^{-1}$ ) inhibited normal root development in all lines examined and delayed germination in all cases except for the curt 1abcd quadruple mutant, which exhibits slower germination than the wild-type ecotypes examined here and naturally produces a lower proportion of viable seeds (Pribil et al. 2018). The combination of terbinafine $(1 \mu \mathrm{M})$ and timentin $\left(200 \mathrm{mg} \mathrm{I}^{-1}\right)$ prevented microbial contamination for 5 days in $100 \%$ of cases, and for 7 days in $90 \%$ of cases (Fig. 3). Henceforth we describe $0.5 \times \mathrm{MS}$ agar containing this combination of terbinafine and timentin as MSTT agar, or MSTT +suc agar when the medium also contains sucrose $(1 \% \mathrm{w} / \mathrm{v})$.

Germination of the four wild-type $A$. thaliana ecotypes and N. tabacum was also examined on MSTT agar
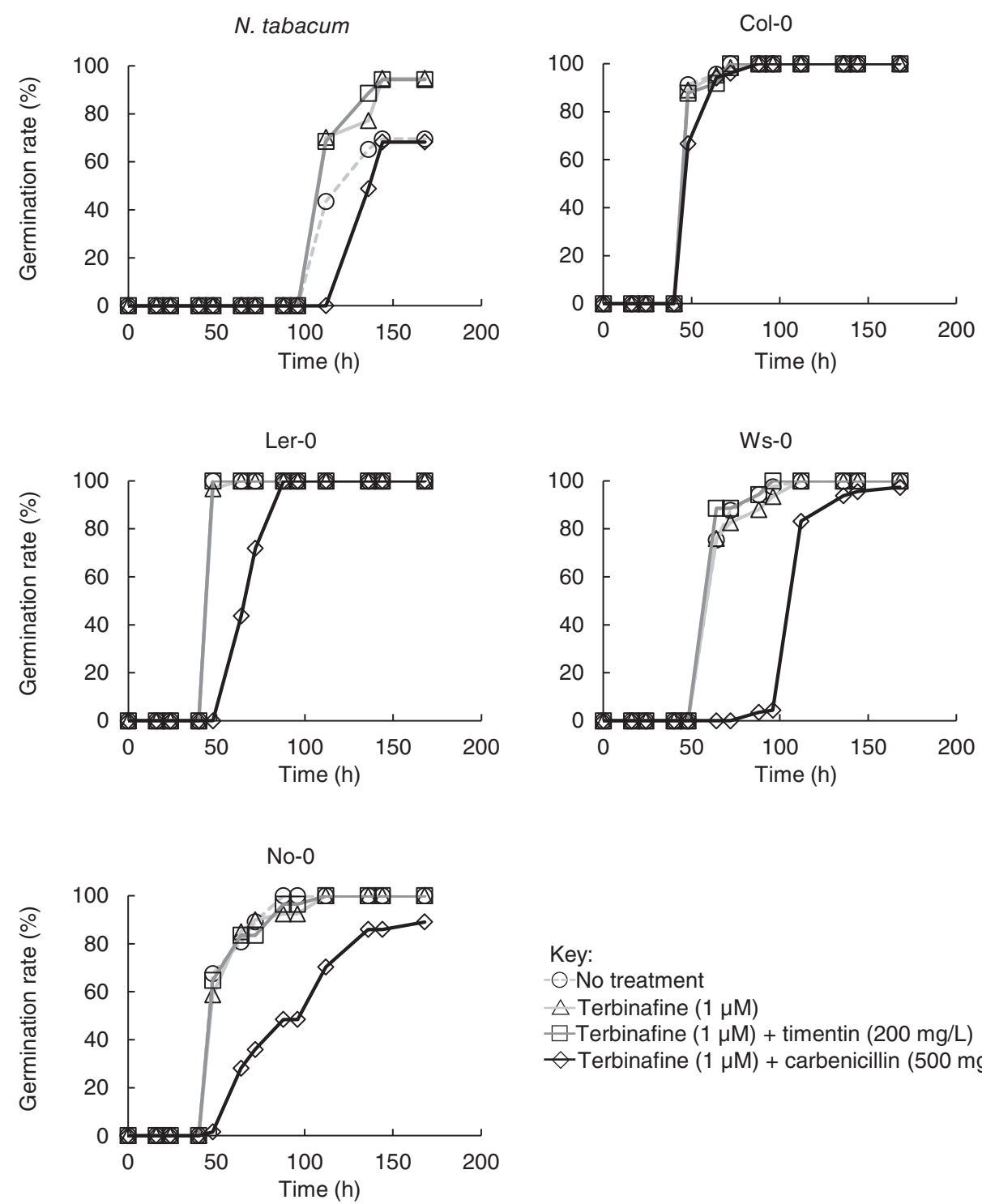

Key:

O-No treatment

$\triangle$ Terbinafine $(1 \mu \mathrm{M})$

$\square$ Terbinafine $(1 \mu \mathrm{M})+$ timentin $(200 \mathrm{mg} / \mathrm{L})$

$\diamond$ Terbinafine $(1 \mu \mathrm{M})+$ carbenicillin $(500 \mathrm{mg} / \mathrm{L})$
Fig. 1. Germination of wild-type seeds in the presence of terbinafine and $\beta$-lactam antibiotics. Non-sterile seeds for Nicotiana tabacum (cv. Petit Havana) and Arabidopsis thaliana ecotypes Columbia (Col$0)$, Landsberg erecta (Ler-0), Wassilewskija (Ws-0), and Nossen (No-0) were sown on $0.5 \times \mathrm{MS}$ agar with added sucrose (1\%, $w / v)$ and different combinations of terbinafine and timentin or carbenicillin (indicated). Germination was monitored by visual inspection and the number of germinated seeds was recorded as a percentage of the total seeds sown on that agar plate. $\mathrm{N}>40$ seeds per plate, except for $N$. tabacum ( $\mathrm{n}>16$ seeds per condition). 
Fig. 2. Germination of Arabidopsis thaliana photosynthetic mutants in the presence of terbinafine and $\beta$-lactam antibiotics. Non-sterile seeds for six $A$. thaliana photosynthetic mutants (atpC1, curt1abcd, pam86, hcf136, npq4 and $p s a L$ ) were sown on $0.5 \times \mathrm{MS}$ agar with added sucrose (1\%, $w / v)$ and different combinations of terbinafine and timentin or carbenicillin (indicated). Germination was monitored by visual inspection and the number of germinated seeds was recorded as a percentage of the total seeds sown on that agar plate $(n>25$ seeds per condition).
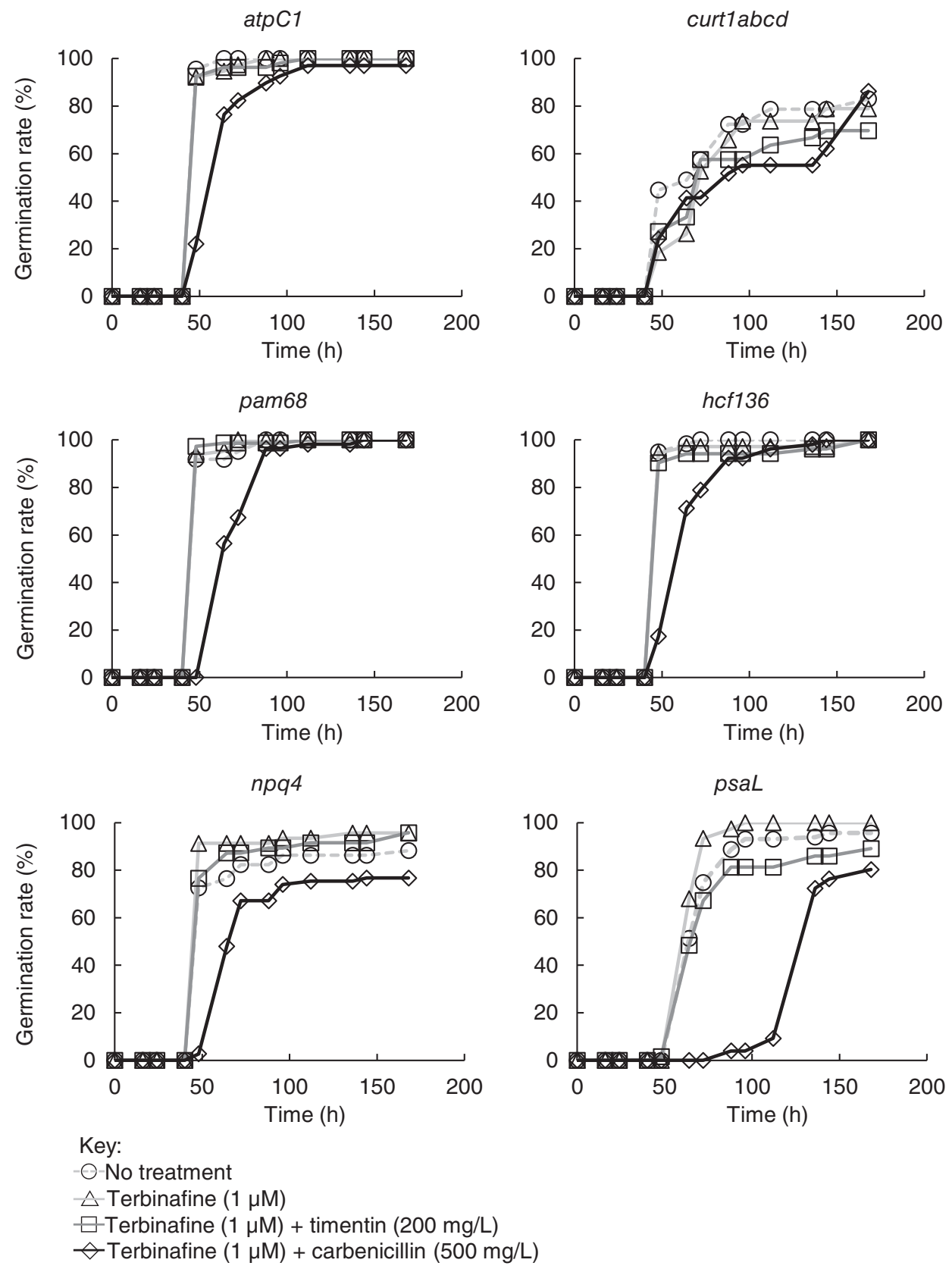

without sucrose. Non-sterile seeds were sown on $0.5 \times$ MS agar or MSTT agar, and germination was unimpeded in all cases (Fig. S2). Contamination emerged on negative control plates (0.5× MS agar) after only $48 \mathrm{~h}$, whereas MSTT agar plates remained free of observable contamination for 1 week (Fig. S3). Microbial contamination emerged on all MSTT agar plates after $184 \mathrm{~h}$. After germination, plants transferred to soil from MSTT and MSTT +suc agar developed into phenotypically normal adult plants, indistinguishable from controls sown directly on soil (Fig. S4).

N. tabacum germination is slightly accelerated by the presence of terbinafine $(1 \mu \mathrm{M})$ and slightly delayed by the presence of sucrose (Fig. 1, Fig. S2, and additional experiments not shown here). We observe these effects consistently when germinating $N$. tabacum but have been unable to find a satisfactory explanation in the scientific literature. 


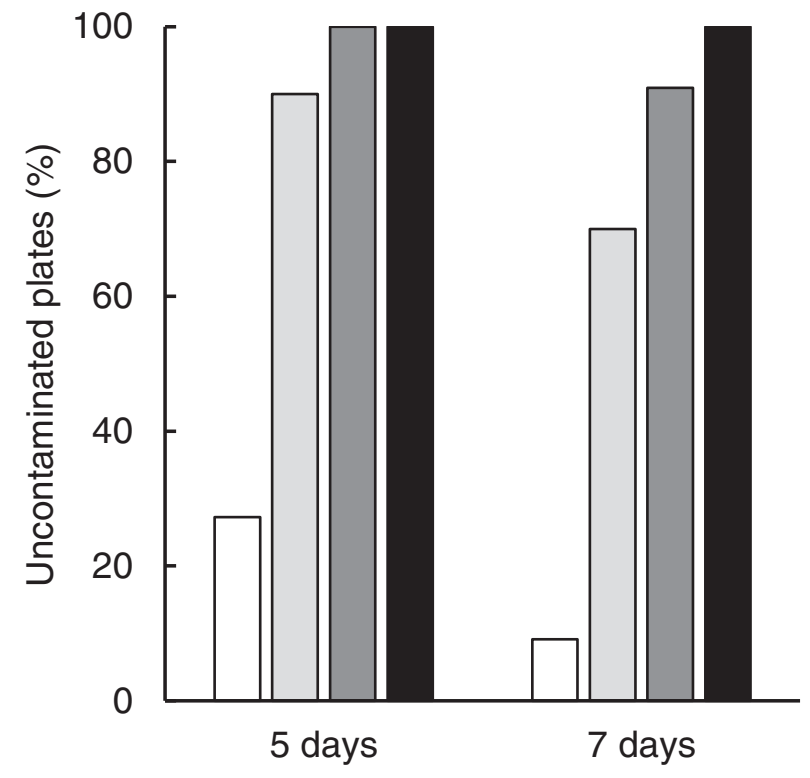

Key:

No treatment

Terbinafine $(1 \mu \mathrm{M})$

Terbinafine $(1 \mu \mathrm{M})+$ timentin $(200 \mathrm{mg} / \mathrm{L})$

Terbinafine $(1 \mu \mathrm{M})+$ carbenicillin $(500 \mathrm{mg} / \mathrm{L})$

Fig. 3. Onset of microbial contamination in the presence of terbinafine and $\beta$-lactam antibiotics. Non-sterile seeds were sown on $0.5 \times$ MS agar with added sucrose $(1 \%, w / v)$ and different combinations of terbinafine and timentin or carbenicillin (indicated). The proportion of agar plates that remained uncontaminated after 5 and 7 days was recorded $(n=11$ agar plates per condition)

\section{Non-sterile screening for Arabidopsis transformants on selective agar}

\section{Screening for nourseothricin resistance and fluorescent protein expression}

Having established that MSTT agar delays microbial contamination without impairing germination, we examined whether this medium would allow us to screen $A$. thaliana genetic transformants without seed sterilization. As a test case, we transfected $A$. thaliana (Col-0) with a green fluorescent protein expression construct, pN_35S/mEGFP: a monomeric enhanced green fluorescent protein (mEGFP) under the control of the cauliflower mosaic virus $35 \mathrm{~S}$ promoter, with a nourseothricin acetyl transferase (nat) selectable marker (Fig. 4A). Non-sterile seeds collected from the $T_{0}$ plant were sown directly onto MSTT agar with added nourseothricin $\left(50 \mathrm{mg} \mathrm{l}^{-1}\right)$. Seeds were stratified directly on the agar plates and then screened using the rapid hypocotyl elongation method that was previously developed for identifying hygromycin B resistance
(Harrison et al. 2006). Briefly, this method involves exposing stratified seeds to light for $6 \mathrm{~h}$ to break dormancy, and then keeping the germinating seedlings in darkness at $22^{\circ} \mathrm{C}$ to promote hypocotyl elongation. Seedlings that express the resistance marker gene grow elongated hypocotyls, whereas non-transformed plants do not (Harrison et al. 2006).

Plates were uncovered after 2 full days of dark treatment (i.e. on the fourth day post-stratification). Individual seedlings with extended hypocotyls were clearly identifiable amongst the majority of seedlings that did not have extended hypocotyls, and fluorescence imaging revealed mEGFP expression (Fig. 4B) in the same seedlings that had extended hypocotyls. No microbial growth was observed and several positive transformants were transferred to soil for propagation. This demonstrated that MSTT agar can be used to limit microbial growth during nourseothricin-based screening, and that nourseothricin is compatible with the hypocotyl elongation rapid screening method. Homozygous transformant lines were identified by repeating the screening procedure with seeds collected from $T_{1}$ and $T_{2}$ plants (Fig. $4 \mathrm{~B}$ ).

We used the same method to identify plants transformed to overexpress the mApple red fluorescent protein (transformed with $\mathrm{pN}$ 35S/mApple) and a nuclear-encoded chloroplast-targeted mCitrine yellow fluorescent protein (pN_35S/CTP-mCitrine) (Fig. S5).

\section{Screening for BASTA resistance and fluorescent protein expression}

We also tested whether non-sterile germination on MSTT agar could be coupled with rapid phosphinothricin (BASTA)-based screening. A. thaliana (Col-0) plants were transfected with the pB_35S/mEGFP expression construct (identical to $\mathrm{pN}$ _35S/mEGFP except that it has a phosphinothricin $\mathrm{N}$-acetyltransferase (bar) selectable marker in place of the nourseothricin acetyl transferase gene) (Fig. 5A). Seeds from the $T_{0}$ plant were sown directly onto MSTT agar with added BASTA $(50 \mu \mathrm{M})$ and stratified as described above. BASTAresistant seedlings were identified using the previously published rapid screening method (Harrison et al. 2006) with minor modifications. Briefly, stratified seeds were exposed to light for $6 \mathrm{~h}$ to break dormancy, and then kept in darkness at $22^{\circ} \mathrm{C}$ for 3 days before transferring to long day growth chamber conditions (see Methods for details). After 2 days in growth chamber conditions, transformed seedlings were clearly identifiable by their dark green expanded cotyledons while non-transformed seedlings exhibited pale unexpanded cotyledons. These phenotypic differences became more pronounced after 3 days in long day conditions. Fluorescence imaging confirmed mEGFP expression in 
Fig. 4. Screening for nourseothricin-resistant transformants with non-sterile seeds. Arabidopsis thaliana (Col0 ) was transfected with a green fluorescent protein expression construct and the resulting seeds were screened for transgene integration in non-sterile conditions on MSTT agar with added nourseothricin (50 $\mathrm{mg} \mathrm{ml}^{-1}$ ). (A) Schematic map of the plasmid used for Agrobacterium-mediated transfection. The T-DNA region is flanked by $L$ and $R$, indicating the left and right border sequences. Monomeric enhanced green fluorescent protein (mEGFP) expression is regulated by the cauliflower mosaic virus $35 \mathrm{~S}$ promoter (CaMV35S). Nourseothricin acetyl transferase (nat) is the selectable marker for plant transformation, and SpcR indicates that the plasmid backbone confers resistance to spectinomycin in bacteria. (B) The screening procedure identified positive transformant $T_{1}$ plants and was repeated to identify homozygous plants in the $\mathrm{T}_{3}$ generation. Transgene integration and expression was confirmed by screening for hypocotyl elongation (indicating nat expression) and mEGFP fluorescence (imaged by illumination with a blue light source and a $530 \mathrm{~nm}$ filter with a $28 \mathrm{~nm}$ bandpass).
A

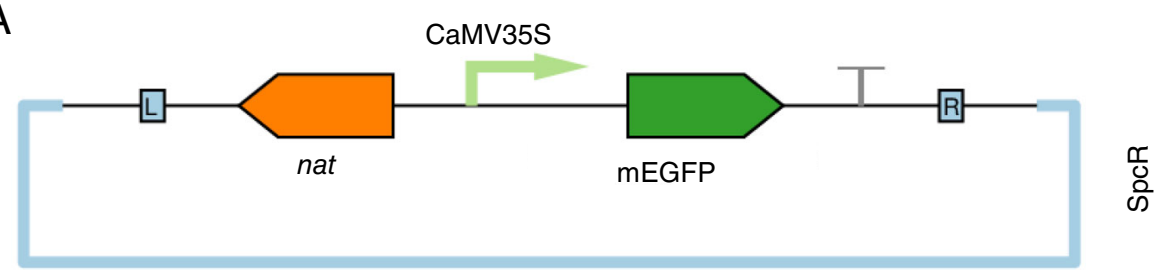

B
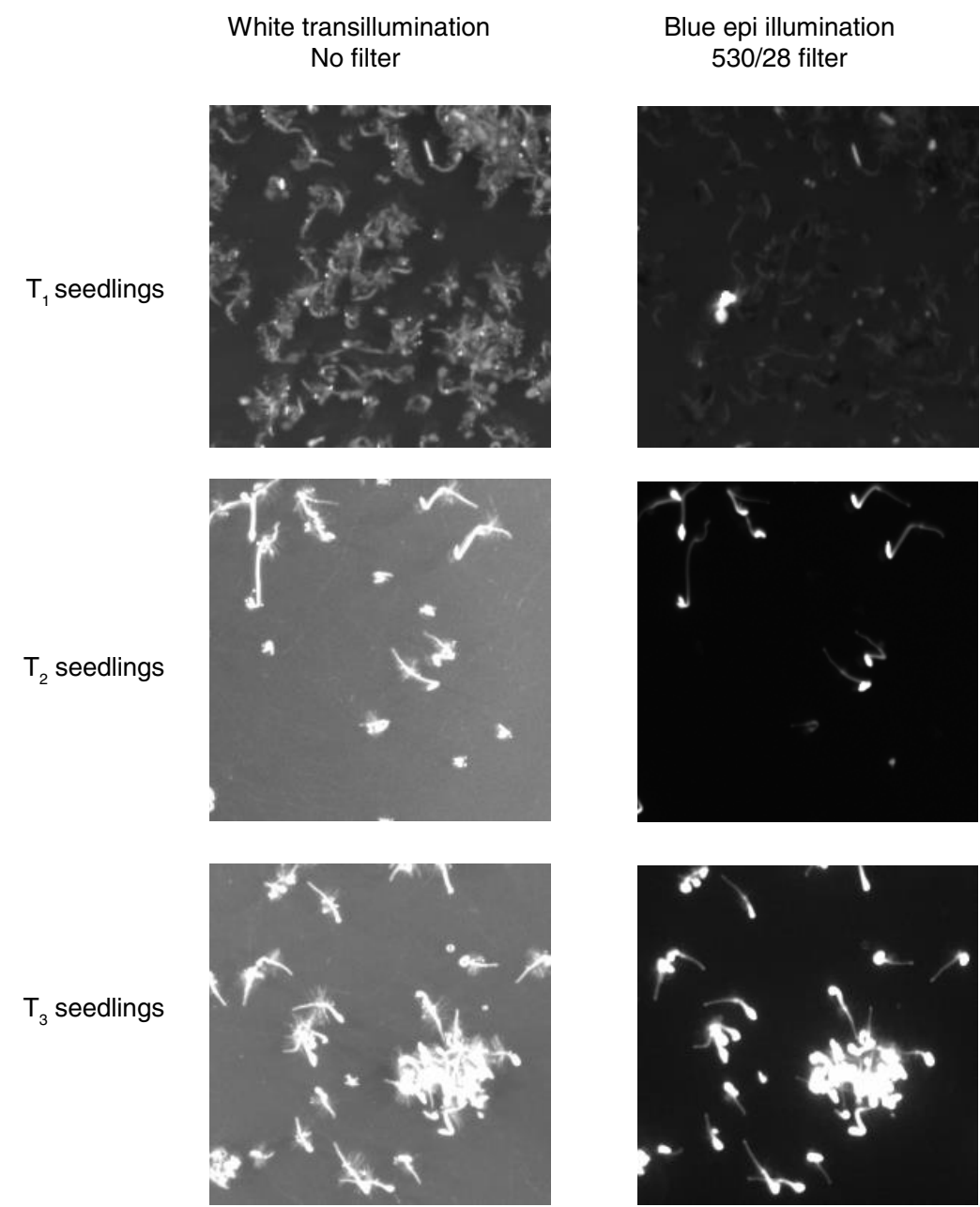

seedlings with green expanded cotyledons (Fig. 5B). No microbial growth was observed during the screening period and multiple positive transformants were identified.

\section{Screening for hygromycin B resistance and identifying mutants produced with CRISPR-Cas9}

In a third test case, we transfected $A$. thaliana (Col-0) with a construct for CRISPR-Cas9-mediated functional knockout of the phytoene desaturase (PDS3) gene, which causes an albino phenotype (Qin et al. 2007). Our plasmid (GS2.1/EC) combined a previously published PDS3-targeting sgRNA (Li et al. 2013) with a Cas9 gene under the control of a previously published egg cell-specific promoter (Wang et al. 2015b). The TDNA region also carried a hygromycin phosphotransferase gene conferring resistance to hygromycin B (Fig. 6A).

Non-sterile seeds collected from $T_{0}$ plants were sown on MSTT+suc agar with hygromycin B $\left(15 \mathrm{mg} \mathrm{l}^{-1}\right)$. Positive transformants were identified on the basis of hypocotyl elongation on the fourth day post-stratification. TDNA integration was confirmed by PCR analysis of leaf tissue (Fig. S6), but all positive transformants in the $T_{1}$ 
A
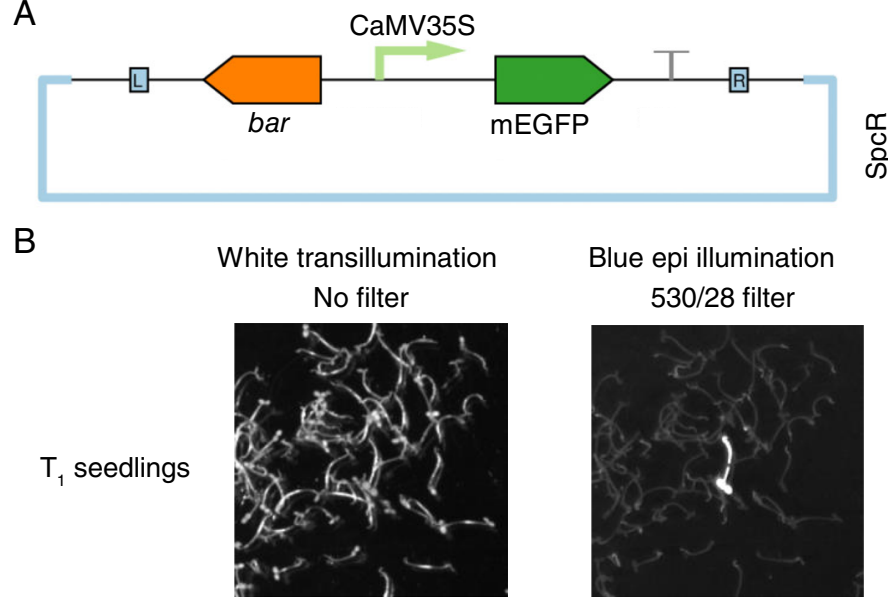

Blue epi illumination $530 / 28$ filter

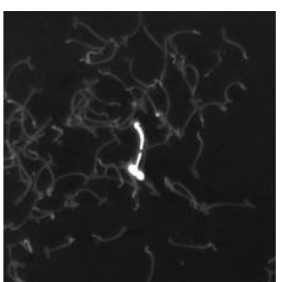

Color photograph

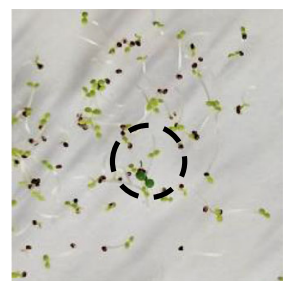

Fig. 5. Screening for BASTA-resistant transformants with non-sterile seeds. Arabidopsis thaliana (Col-0) was transfected with a green fluorescent protein expression construct and the resulting seeds were screened for transgene integration in non-sterile conditions on MSTT agar with added BASTA (50 $\mu \mathrm{M}$ ). (A) Schematic map of the plasmid used for agrobacterium-mediated transfection. The T-DNA region is flanked by $L$ and $R$, indicating the left and right border sequences. Monomeric enhanced green fluorescent protein (mEGFP) expression is regulated by the cauliflower mosaic virus $35 \mathrm{~S}$ promoter (CaMV35S). Phosphinothricin $\mathrm{N}$-acetyl transferase (bar) is the selectable marker for plant transformation, and SpcR indicates that the plasmid backbone confers resistance to spectinomycin in bacteria. (B) The screening procedure identified positive transformant $T_{1}$ plants. Transgene integration and expression was confirmed by screening for cotyledon expansion and greening (indicating bar expression) and mEGFP fluorescence (imaged by illumination with a blue light source and a $530 \mathrm{~nm}$ filter with a $28 \mathrm{~nm}$ bandpass). The positive transformant, distinguishable by its larger, dark green cotyledons, is circled with a dashed line in the color photograph panel.

generation had green cotyledons indicating that any post-transfection CRISPR-Cas9 activity during seed development had not resulted in homozygous pds3 mutants.

Non-sterile seeds collected from one $T_{1}$ plant were sown on MSTT+suc agar containing hygromycin B $\left(15 \mathrm{mg} \mathrm{l}^{-1}\right)$. Approximately 1500 seeds were sown on a single $12 \times 12 \mathrm{~cm}$ agar plate. Approximately $75 \%$ of seedlings were resistant to hygromycin $B$, and on the fifth day post-stratification (i.e. after cotyledon greening) three albino pds3 mutants were identified (Fig. 6B). The three albino seedlings were confirmed as independent pds3 mutants using Sanger sequencing (Fig. S7).

\section{Discussion}

When producing new $A$. thaliana transgenic lines, experimental throughput is partly limited by the transformant screening process. In particular, the seed sterilization step is time consuming and can cause seed mortality (Lindsey et al. 2017). Alternative screening methods that negate the need for seed sterilization have been developed, each with advantages and disadvantages.

Conventional selection for BASTA resistance (conferred by bar, the phosphinothricin $\mathrm{N}$-acetlytransferase gene) (D'Halluin et al. 1992) involves spraying the aerial parts of germinated seedlings and can be performed in non-sterile conditions with seeds sown directly on soil at relatively high densities. The disadvantage of this approach is the time required to identify positive transformants: typically at least three spray applications are spread across 3 weeks (Leclere and Bartel 2001). Additionally, the use of BASTA is restricted in some countries due to concerns surrounding neurotoxicity linked to BASTA ingestion (Hack et al. 1994, Watanabe and Sano 1998, Mao et al. 2011).

A more modern approach to avoiding seed sterilization is to use a fluorescent protein with seed-specific expression as the marker gene (Stuitje et al. 2003, Shimada et al. 2010). Accumulation of the fluorescent protein in transformed seeds can be observed visually with a suitable light source and filter combination (a fluorescence microscope is typically used). Visual screening for a co-expressed fluorescent protein avoids the need for sterilization, and only seeds with active transgene expression are sown on soil. This is an excellent approach for avoiding seed mortality and reducing the number of plants that need to be grown in a screening campaign, but this approach is still best suited to scenarios involving relatively few transgenic lines due to the labour involved in screening seeds under a fluorescence microscope.

The method we present here provides another pragmatic option for avoiding seed sterilization. When paired with rapid screening methods, seeds sown on MSTT or MSTT+suc agar could be screened for resistance to 
A

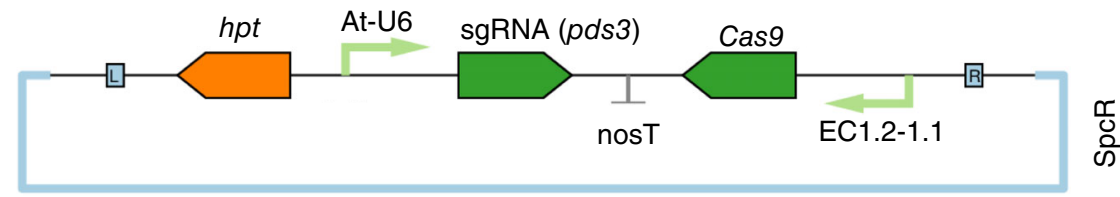

B

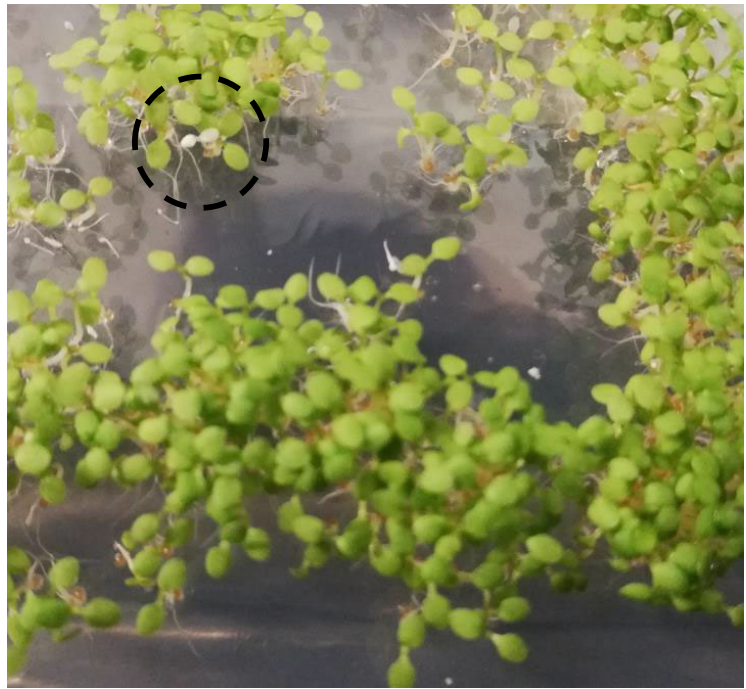

Fig. 6. Screening for a CRISPR-Cas9-mediated pds3 mutant phenotype with non-sterile seeds. Arabidopsis thaliana (Col-0) was transfected with a CRISPR-Cas9 plasmid targeting mutation of the phytoene desaturase, PDS3. Homozygous pds3 mutants were obtained by screening seeds in nonsterile conditions on MSTT+suc agar with added hygromycin $B\left(15 \mathrm{mg} \mathrm{l}^{-1}\right)$. (A) Schematic map of the plasmid used for agrobacterium-mediated transfection. The T-DNA region is flanked by $L$ and $R$, indicating the left and right border sequences. A single guide RNA (sgRNA) targeting PDS3 is regulated by the $A$. thaliana U6 polymerase III promoter (At-U6). The Cas9 gene is regulated by an egg cell-specific promoter (EC1.2-1.1). The hygromycin phosphotransferase (hpt) is the selectable marker for plant transformation, and SpcR indicates that the plasmid backbone confers resistance to spectinomycin. (B) Non-sterile seeds were screened on MSTT+suc agar with hygromycin B. Homozygous pds3 knockout mutants were identifiable in the $T_{2}$ generation by their characteristic albino phenotype. An example pds3 mutant on day five post-stratification is indicated inside the dashed circle.

nourseothricin, hygromycin B or BASTA in as few as 4-5 days after stratification. This approach allowed us to curate homozygous $T_{3}$ transgenic lines and identify CRISPR-Cas9-mediated pds3 knockout mutants without the need for seed sterilization at any stage. It is possible that the transgene selection reagents also contribute to the antimicrobial effect when used in combination with terbinafine and timentin, but nourseothricin and hygromycin B were not sufficient to prevent contamination when used alone (data not shown).

The MSTT formulation that we developed can be used to delay the onset of microbial growth or decrease the likelihood of contaminants becoming established, but it cannot be used to maintain sterile conditions outright. Therefore, we can only recommend its use with species that germinate quickly (i.e. in less than a week). Additionally, all seeds used in this study were produced in growth chamber conditions; our method may not be suitable for use with field-grown seeds that could be expected to have a greater microbial burden.
Although MSTT and MSTT+suc agar did not negatively affect germination of any of the seeds examined in this study, we only validated these media for use in screening laboratory-grown seeds for transgene insertion. It is unknown whether exposure to sub-inhibitory concentrations of terbinafine and timentin may trigger any responses in Arabidopsis that would make these media unsuitable for physiological studies.

When developing the MSTT formulation, we identified terbinafine as a candidate antifungal reagent from an earlier study that investigated squalene metabolism in Arabidopsis (Laranjeira et al. 2015). We also tested miconazole $\left(20 \mathrm{mg} \mathrm{l}^{-1}\right)$ on the basis that it had been used to prevent fungal overgrowth in explant tissue culture of a number of crop species (Tynan et al. 1993), but miconazole was $100 \%$ lethal to all of our Arabidopsis lines at this concentration (data not shown). It is possible that miconazole would be useful at lower concentrations but we did not pursue this further given our immediate success with terbinafine. 
While the inhibitory effect of terbinafine on squalene epoxidase in plants has been characterized, the effects of $\beta$-lactam antibiotics on plants are not fully understood. Peptidoglycan biosynthesis is retained in moss chloroplasts but is absent from vascular plants (Hirano et al. 2016), and it is generally assumed that $\beta$-lactam antibiotics do not affect the chloroplasts of higher plants (Reski 2009). Carbenicillin (500 $\mathrm{mg} \mathrm{l}^{-1}$ ) has been described in the literature as beneficial for eliminating $\beta$-lactam-sensitive Agrobacterium strains from transfected Arabidopsis and tobacco tissue culture (Lin et al. 1995), and carbencillin concentrations between 100 and $500 \mathrm{mg} \mathrm{I}^{-1}$ have also been recommended for use in solid media when screening $\mathrm{T}_{1}$ transgenic Arabidopsis seeds after floral dip transformation (Zhang et al. 2006, Cold Spring Harbor Protocols 2010, Yan et al. 2015). We initially planned to use a high concentration of carbenicillin $\left(500 \mathrm{mg} \mathrm{I}^{-1}\right)$ on the basis that $\beta$-lactamase enzymes are secreted by many environmental bacteria and some commonly used laboratory strains of Agrobacteria (Ogawa and Mii 2004). However, carbenicillin and penicillin were recently reported to impair root elongation in A. thaliana at concentrations between 100 and $1000 \mathrm{mg} \mathrm{l}^{-1} \mathrm{~L}$ (Gudiño et al. 2018). As an alternative to carbenicillin, we considered timentin on the basis that a lower concentration of timentin should provide a similar protective effect due to the presence of a $\beta$-lactamase inhibitor (clavulanic acid) in the timentin formulation. We observed that carbenicillin (500 $\mathrm{mg} \mathrm{l}^{-1}$ ) did not cause seed mortality but delayed germination and prevented root elongation, whereas timentin (200 $\left.\mathrm{mg}^{-1}\right)$ had no observable effect on Arabidopsis germination and growth.

In conclusion, timentin and terbinafine added to $0.5 \times$ MS agar delay the onset of microbial contamination and do not inhibit germination of $A$. thaliana or $N$. tabacum. The inhibition of microbial growth is sufficient to allow selection of transgenic plants from non-sterile seeds, avoiding the time-consuming seed sterilization step and minimizing seed mortality.

We believe that our method provides a useful alternative approach to simplifying Arabidopsis transformant screening. It requires minimal labour and can be used with rapid screening methods for BASTA, nourseothricin and hygromycin $B$ resistance. When nourseothricin or hygromycin B are used as the transgene selection reagents, seeds can be sown at high densities and screened on the basis of hypocotyl elongation. For rapid BASTA resistance screening, seeds must be sown at low densities to allow the user to distinguish between green (positive transformant) and pale yellow (wild-type) cotyledons, and we anticipate that this would also be the case for kanamycin-based rapid screening (Harrison et al. 2006).

\section{Author contributions}

J.B.Y.H.B. conceived of the concept, designed and executed the experiments, and wrote the manuscript. G.B.G. validated the use of MSTT agar for BASTA-based screening. M.P. contributed to experimental design and writing the manuscript.

Acknowledgements - We thank Dr. Omar SandovalIbañez (Max Planck Institute of Molecular Plant Physiology, Germany) and Dr. Lars Scharff (University of Copenhagen, Denmark) for their feedback on the manuscript. This project has received funding from the European Union's Horizon 2020 research and innovation programme under Marie Skłodowska Curie Actions Individual Fellowship grant agreement No. 752430 (awarded to JBYHB).

\section{Data availability statement}

Plasmids created for this study are available at www. addgene.org using the reference numbers described in the text and summarized online in Supporting Information Fig S2. Raw data are available at the following URL: https://www.dropbox.com/s/19ze4qxcthgq0wo/ Behrendorff\%20et $\% 20$ al\%20antimicrobials $\% 20$ raw $\%$ 20data.zip?dl=0.

\section{References}

Andargie M, Li J (2016) Arabidopsis thaliana: a model host plant to study plant-pathogen interaction using rice false smut isolates of Ustilaginoidea virens. Front Plant Sci 7: 192. https://doi.org/10.3389/fpls.2016.00192

Armbruster U, Labs M, Pribil M, Viola S, Xu W, Scharfenberg M, Hertle AP, Rojahn U, Jensen PE, Rappaport F, Joliot P, Dormann P, Wanner G, Leister D (2013) Arabidopsis CURVATURE THYLAKOID1 proteins modify THYLAKOID architecture by inducing membrane curvature. Plant Cell 25: 2661-2678. https://doi.org/10. 1105/tpc. 113.113118

Armbruster U, Zühlke J, Rengstl B, Kreller R, Makarenko E, Rühle T, Schünemann D, Jahns P, Weisshaar B, Nickelsen J, Leister D (2010) The Arabidopsis thylakoid protein PAM68 is required for efficient D1 biogenesis and photosystem II assembly. Plant Cell 22: 3439-3460. https://doi.org/10.1105/tpc.110.077453

Bechtold N, Pelletier G (1998) In planta Agrobacteriummediated transformation of adult Arabidopsis thaliana plants by vacuum infiltration. In: Clifton NJ (ed) Methods in Molecular Biology. Springer Berlin Heidelberg, Berlin, Heidelberg, pp 259-266 
Behrendorff JBYH, Sandoval-Ibañez OA, Sharma A, Pribil M (2019) Membrane-bound protein scaffolding in diverse hosts using thylakoid protein CURT1A. ACS Synth Biol 8: 611-620. https://doi.org/10.1021/acssynbio. 8 b00418

Bhatia S, Densmore D (2013) Pigeon: a design visualizer for synthetic biology. ACS Synth Biol 2: 348-350. https://doi. org/10.1021/sb400024s

Chung MH, Chen MK, Pan SM (2000) Floral spray transformation can efficiently generate Arabidopsis transgenic plants. Transgenic Res 9: 471-476

Cold Spring Harbor Protocols (2010) Selection Plates for Transgenic Arabidopsis. Cold Spring Harbour Press. Cold Spring Harbor, NY. https://doi.org/10.1101/pdb. rec12144. Accessed 15 Nov 2019

D'Halluin K, De Block M, Denecke J, et al. (1992) The bar gene as selectable and screenable marker in plant engineering. Methods Enzymol 216: 415-426. https://doi. org/10.1016/0076-6879(92)16038-I

Dal Bosco C, Lezhneva L, Biehl A, et al. (2004) Inactivation of the chloroplast ATP synthase gamma subunit results in high non-photochemical fluorescence quenching and altered nuclear gene expression in Arabidopsis thaliana. J Biol Chem 279: 1060-1069. https://doi.org/10.1074/jbc. M308435200

Ellis RJ (1969) Chloroplast ribosomes: stereospecificity of inhibition by chloramphenicol. Science 163: 477-478. https://doi.org/10.1126/science.163.3866.477

Gan HM, Savka MA (2018) One more decade of Agrobacterium taxonomy. In: Gelvin S (ed) Agrobacterium Biology. Springer, Cham, pp 1-14

Gibson DG, Young L, Chuang R-Y, Venter JC, Hutchison CA III, Smith HO (2009) Enzymatic assembly of DNA molecules up to several hundred kilobases. Nat Methods 6: 343-345. https://doi.org/10.1038/nmeth.1318

Gudiño ME, Blanco-Touriñán N, Arbona V, et al. (2018) $\beta$-Lactam antibiotics modify root architecture and indole glucosinolate metabolism in Arabidopsis thaliana. Plant Cell Physiol 59: 2086-2098. https://doi.org/10.1093/pcp/ pcy 128

Hack R, Ebert E, Ehling G, Leist K-H (1994) Glufosinate ammonium - some aspects of its mode of action in mammals. Food Chem Toxicol 32: 461-470. https://doi. org/10.1016/0278-6915(94)90043-4

Harrison SJ, Mott EK, Parsley K, Aspinall S, Gray JC, Cottage $A$ (2006) A rapid and robust method of identifying transformed Arabidopsis thaliana seedlings following floral dip transformation. Plant Methods 2: 19. https://doi. org/10.1186/1746-4811-2-19

Hirano T, Tanidokoro K, Shimizu Y, Kawarabayasi Y, Ohshima T, Sato M, Tadano S, Ishikawa H, Takio S, Takechi K, Takano H (2016) Moss chloroplasts are surrounded by a peptidoglycan wall containing D-amino acids. Plant Cell 28: 1521-1532. https://doi.org/10.1105/ tpc.16.00104
Jelenska J, Tietze E, Tempé J, Brevet J (2000) Streptothricin resistance as a novel selectable marker for transgenic plant cells. Plant Cell Rep 19: 298-303. https://doi.org/10. 1007/s002990050016

Karimi M, Inzé D, Depicker A (2002) GATEWAYTM vectors for agrobacterium-mediated plant transformation. Trends Plant Sci 7: 193-195. https://doi.org/10.1016/S13601385(02)02251-3

Laranjeira S, Amorim-Silva V, Esteban A, Arró M, Ferrer A, Tavares RM, Botella MA, Rosado A, Azevedo H (2015) Arabidopsis squalene epoxidase 3 (SQE3) complements SQE1 and is important for embryo development and bulk squalene epoxidase activity. Mol Plant 8: 1090-1102. https://doi.org/10.1016/J.MOLP.2015.02.007

Leclere S, Bartel B (2001) A library of Arabidopsis 35S-cDNA lines for identifying novel mutants. Plant Mol Biol 46: 695-703

Li J-F, Norville JE, Aach J, McCormack M, Zhang D, Bush J, Church GM, Sheen J (2013) Multiplex and homologous recombination-mediated genome editing in Arabidopsis and Nicotiana benthamiana using guide RNA and Cas9. Nat Biotechnol 31: 688-691. https://doi.org/10.1038/nbt. 2654

Li X-P, Björkman O, Shih C, Grossman AR, Rosenquist M, Jansson S, Niyogi KK (2000) A pigment-binding protein essential for regulation of photosynthetic light harvesting. Nature 403: 391-395. https://doi.org/10.1038/35000131

Lin J-J, Assad-Garcia N, Kuo J (1995) Plant hormone effect of antibiotics on the transformation efficiency of plant tissues by Agrobacterium tumefaciens cells. Plant Sci 109: 171-177. https://doi.org/10.1016/0168-9452(95) 04168-T

Lindsey BE, Rivero L, Calhoun CS, et al. (2017) Standardized method for high-throughput sterilization of Arabidopsis seeds. J Vis Exp. 128: e56587. https://doi.org/10.3791/ 56587

Lunde C, Jensen PE, Haldrup A, Knoetzel J, Scheller HV (2000) The PSI-H subunit of photosystem I is essential for state transitions in plant photosynthesis. Nature 408: 613-615. https://doi.org/10.1038/35046121

Mao Y, Wang J-D, Hung D-Z, Deng JF, Yang CC (2011) Hyperammonemia following glufosinate-containing herbicide poisoning: a potential marker of severe neurotoxicity. Clin Toxicol 49: 48-52. https://doi.org/10. 3109/15563650.2010.539184

Martinez-Trujillo M, Limones-Briones V, Cabrera-Ponce JL, Herrera-Estrella L (2004) Improving transformation efficiency of Arabidopsis thaliana by modifying the floral dip method. Plant Mol Biol Report 22: 63-70. https://doi. org/10.1007/BF02773350

Meurer J, Plücken H, Kowallik KV, Westhoff P (1998) A nuclear-encoded protein of prokaryotic origin is essential for the stability of photosystem II in Arabidopsis thaliana. EMBO J 17: 5286-5297. https://doi.org/10.1093/emboj/ 17.18.5286 
Mulo P, Pursiheimo S, Hou C-X, Tyystjärvi T, Aro EM (2003) Multiple effects of antibiotics on chloroplast and nuclear gene expression. Funct Plant Biol 30: 1097. https://doi. org/10.1071/FP03149

Murashige T, Skoog F (1962) A revised medium for rapid growth and bio assays with tobacco tissue cultures. Physiol Plant 15: 473-497. https://doi.org/10.1111/j. 1399-3054.1962.tb08052.x

Ogawa Y, Mii M (2004) Screening for highly active $\beta$-lactam antibiotics against Agrobacterium tumefaciens. Arch Microbiol 181: 331-336. https://doi.org/10.1007/s00203004-0650-z

Pribil M, Sandoval-Ibáñez O, Xu W, et al. (2018) CURT1-mediated thylakoid plasticity is required for finetuning photosynthesis and plant fitness. Plant Physiol 176: 2351-2364. https://doi.org/10.1104/pp.17.00863

Qin G, Gu H, Ma L, Peng Y, Deng XW, Chen Z, Qu LJ (2007) Disruption of phytoene desaturase gene results in albino and dwarf phenotypes in Arabidopsis by impairing chlorophyll, carotenoid, and gibberellin biosynthesis. Cell Res 17: 471-482. https://doi.org/10.1038/cr.2007.40

Reski R (2009) Challenges to our current view on chloroplasts. Biol Chem 390: 731-738. https://doi.org/10. 1515/BC.2009.089

Ryder NS (1992) Terbinafine: mode of action and properties of the squalene epoxidase inhibition. Br J Dermatol 126: 2-7. https://doi.org/10.1111/j.1365-2133.1992.tb00001.x

Shimada TL, Shimada T, Hara-Nishimura I (2010) A rapid and non-destructive screenable marker, FAST, for identifying transformed seeds of Arabidopsis thaliana. Plant J 61: 519-528. https://doi.org/10.1111/j.1365-313X. 2009.04060.x

Stuitje AR, Verbree EC, Van Der Linden KH, et al. (2003) Seed-expressed fluorescent proteins as versatile tools for easy (co)transformation and high-throughput functional genomics in Arabidopsis. Plant Biotechnol J 1: 301-309. https://doi.org/10.1046/j.1467-7652.2003.00028.x

Tynan JL, Conner AJ, Macknight RC, Poulter RTM (1993) Miconazole: an effective antifungal agent for plant tissue culture. Plant Cell Tiss Org Cult 32: 293-301. https://doi. org/10.1007/BF00042292

Wang X, Ryu D, Houtkooper RH, Auwerx J (2015a) Antibiotic use and abuse: a threat to mitochondria and chloroplasts with impact on research, health, and environment. Bioessays 37: 1045-1053. https://doi.org/ 10.1002/bies.201500071

Wang Z-P, Xing H-L, Dong L, Zhang HY, Han CY, Wang XC, Chen QJ (2015b) Egg cell-specific promoter-controlled CRISPR/Cas9 efficiently generates homozygous mutants for multiple target genes in Arabidopsis in a single generation. Genome Biol 16: 144. https://doi.org/10. 1186/s13059-015-0715-0

Watanabe T, Sano T (1998) Neurological effects of glufosinate poisoning with a brief review. Hum Exp Toxicol 17: 35-39
Yan L, Wei S, Wu Y, Hu R, Li H, Yang W, Xie Q (2015) Highefficiency genome editing in Arabidopsis using YAO promoter-driven CRISPR/Cas9 system. Mol Plant 8: 1820-1823. https://doi.org/10.1016/j.molp.2015.10.004

Zhang X, Henriques R, Lin S-S, Niu QW, Chua NH (2006) Agrobacterium-mediated transformation of Arabidopsis thaliana using the floral dip method. Nat Protoc 1 : 641-646. https://doi.org/10.1038/nprot.2006.97

\section{Supporting information}

Additional supporting information may be found online in the Supporting Information section at the end of the article.

Fig. S1. Terbinafine as an antifungal reagent. Non-sterile seeds were sown on $0.5 \times$ MS agar with added sucrose $(1 \%, w / v)$ and different concentrations of terbinafine (indicated). (A) Germination onset is defined as the emergence of cotyledons from the first germinating seeds on each agar plate. (B) Each marker $(x)$ indicates the time at which microbial contamination emerged on individual agar plates. The overall frequency of contamination is summarised at the top of the plot.

Fig. S2. Germination of non-sterile wild-type seeds on MSTT agar. Non-sterile seeds of $N$. tabacum and four A. thaliana ecotypes were sown on $0.5 \times \mathrm{MS}$ agar (०) or MSTT agar $(\square)$. Plates were observed twice per day and the proportion of germinated seeds was recorded. Germination was defined as cotyledon emergence.

Fig. S3. Germination of wild type seeds on $0.5 \times \mathrm{MS}$ and MSTT agar. Non-sterile wild-type seeds for $A$. thaliana ecotypes Columbia (Col 0), Landsberg erecta (Ler 0), Wassilewskija (Ws 0) and Nossen (No 0), and for N. tabacum were germinated on either $0.5 \times \mathrm{MS}$ agar or MSTT agar. Plates were photographed on the seventh day after stratification. A variety of microbial growth is visible on the $0.5 \times \mathrm{MS}$ agar plates, whereas no microbial growth was observed on MSTT agar.

Fig. S4. Adult plants germinated on soil or MSTT agar. Wild-type A. thaliana accessions and N. tabacum were germinated on soil or on MSTT agar. Seeds on MSTT agar were stratified directly on the agar plates, while soil germinated seeds were stratified on wet filter paper and then transferred to soil. Seedlings germinated on MSTT agar were transferred to soil 5 days post-stratification. Adult plants were photographed 20 days post-stratification, except for Ler-0 plants which were photographed 14 days post stratification.

Fig. S5. Verification of transgene expression with fluorescence imaging. Positive transformants for nuclear 
encoded chloroplast targeted mCitrine expression (A) and mApple expression (B) were verified with fluorescence imaging.

Fig. S6. Verification of Cas9 presence in positive GS2.1/EC transformant lines. Six independent $T_{1}$ plants were identified that showed resistance to hygromycin B. Leaf tissue was sampled for PCR with primers specific to a 1059 bp section of the Cas9 gene. The negative control is tissue from untransformed A. thaliana Col-0 grown on soil. Agarose gel electrophoresis of the PCR products is shown. Gel layout: $1 \mathrm{~kb}$ Plus DNA ladder (ThermoFisher Scientific Cat. no. 10787018), $T_{1}$ plants 1-6, untransformed A. thaliana Col 0 (negative control).

Fig. S7. Verification of pds3 knockouts. CRISPRCas9-mediated knockout of the pds3 gene was performed by stable transfection of $A$. thaliana Col-0 with the GS2.1/ EC construct. The targeted region of $p d s 3$ from three albino A. thaliana mutants was sequenced. All three mutants are independent knockout lines. pds3-2 is homozygous for a single nucleotide insertion, while $p d s 3-1$ and pds3-3 appear to have heterozygous knockout mutations (i.e. different mutations on each chromosome), indicated by mixed chromatogram peaks after the apparent Cas9 cut site. 